\title{
Tropical sprue: case report with review of literature
}

\author{
Robinson $\mathrm{W}^{1}$, Bhattacharya $\mathrm{B}^{2}$ \\ ${ }^{1}$ Clinical Gastroenterology Fellow, Ohio State Medical Center, Division of Gastroenterology, Hepatology and Nutrition, USA \\ ${ }^{2}$ Surgical and Gastrointestinal Pathologist, Caris Diagnostics, Part of Miraca Holdings, Inc, Phoenix, USA
}

\author{
Keywords: \\ Coeliac disease; \\ Coeliac sprue; \\ Megaloblastic anemia; \\ Tropical sprue
}

\begin{abstract}
Tropical sprue is an important cause of malabsorption in adults and in children in South Asia. Investigations to exclude specific infective, immunological or inflammatory causes are important before considering tropical sprue as a diagnosis. Malabsorption disorders of the small intestine can be a source of great debilitation to the patient, and a diagnostic challenge to the pathologist. This article presents clinicopathological findings of a non coeliac tropical sprue in a 66-year-old male and discusses the histological and clinical information that can help differentiate coeliac from non coeliac sprue.
\end{abstract}

\section{INTRODUCTION}

The gastrointestinal tract, especially the small intestine is an important organ where most nutrients including vitamins, iron, protein, carbohydrate and fat get absorbed. The increased surface area of the small intestine due to plica circularis, villi and microvilli is specially suited for this role. Intestinal malabsorption can arise from a variety of causes (Table 1$){ }^{1}$

Tropical sprue (TS) is an acquired disease of unknown etiology, characterized by malabsorption, multiple nutritional deficiencies and mucosal abnormalities in the small bowel. The definition of the various clinical syndromes that are together termed tropical sprue is still controversial. Baker and Klipstein ${ }^{2}$ suggested that the diagnosis of tropical sprue should be made only when there is malabsorption of two or more unrelated nutrient groups (eg, fat and carbohydrate), and after other known causes of malabsorption have been excluded.

\section{Correspondence:}

Dr. Baishali Bhattacharya, $M D, M P H$

Caris Diagnostics Part of Miraca Holdings, Inc.

4207 East Cotton Center Blvd.

Phoenix AZ 85040, USA

Email:baishalib@hotmail.com
The pathology specimens consist mostly of biopsies of the duodenum or proximal jejunum, or resections of the small intestine. The various compartments that need to be analyzed include the lumen, villous architecture, lamina propria, submucosa, and muscularis propris. In this report, we present the clinicopathological findings of a non coeliac TS in a 66 year old male and discuss the histological and clinical information that can help differentiate coeliac from non coeliac sprue.

\section{CASE REPORT}

A 66-year-old male with an unremarkable past medical history presented with two weeks of non-bloody diarrhea and worsening fatigue. The acute symptoms had been preceded by 2 months of anorexia and a 10 pounds weight loss. He denied abdominal pain, nausea, vomiting, fevers, sick contacts, or recent travel. He had immigrated to the United States from the Dominican Republic over 20 years ago and briefly returned 5 years prior to presentation. His medications included only an anti-diarrheal (Imodium) drug, taken over the preceding two weeks.

Physical examination revealed a fatigued male with normal vital signs. The examination showed $1-2+$ pretibial pitting 


\section{Table 1: Causes of tropical malabsorption ${ }^{1}$}

- Coeliac disease

- Infections such as Giardia, Mycobacterium avium, intestinal tuberculosis, cryptosporidia, Cyclospora, Isospora, Helminths, Leishmania

- Lactose intolerance (biopsy finding is normal)

- Immunodeficiency and HIV/AIDS related enteropathy, including IgA deficiency, common variable immunodeficiency (HIV/AIDS enteropathy is diagnosed when other infections are excluded)

- Tropical sprue

- Small bowel bacterial overgrowth (caused by anaerobic bacterial overgrowth due to stasis, diabetic neuropathy, bowel resections, scleroderma etc)

- Crohn's enteritis

- Autoimmune enteropathy (children, anti-enterocyte antibodies)

- Chronic pancreatitis

- Malignancy such as carcinoma, lymphoma

\section{Table 2: Biopsy protocol for diagnosis of malabsortion}

Multiple biopsies from 2nd, 3rd part duodenum and proximal jejunum if possible

Importance of proper biopsy orientation

Diagnosis of coeliac disease is based on biopsy findings, serology and response to Gluten free diet.

Normal intraepithelial T lymphocytes: $<40 / 100$ enterocytes with $<12$ in the villous tip

Normal villous: crypt ratio: 3-5:1

Beware of the followings:

- Duodenal bulb often shows peptic change and can be blunted

- Terminal ileum peyers patches can appear blunted with intraepithelial lymphocytes in the overlying epithelium

- Beware of thick or tangential sections

- Increased duodenal IELs without villous blunting

»Coeliac sprue including treated and latent sprue

» Helicobacter pylori infection in the stomach

» Non steroidal anti-inflammatory medications

» Infection

» Idiopathic inflammatory bowel disease (Crohn's disease)

» Tropical sprue

„ Food allergy

»Connective tissue disorder

» Morbid obesity edema and conjunctival pallor. The abdominal and rectal examinations were unremarkable. Initial blood test identified a macrocytic anemia with hemoglobin $6.2 \mathrm{~g} / \mathrm{dl}$, MCV $108.5 \mathrm{fL}$, WBC 3500/cumm, and platelets 154,000/cumm. Additional blood tests revealed severe deficiencies in Vitamin $\mathrm{B}_{12}: 31 \mathrm{pg} /$ $\mathrm{ml}$ (normal: $200-600 \mathrm{pg} / \mathrm{ml}$ ) and folate: $102 \mathrm{ng} / \mathrm{ml}$ (normal red cell folate: $160-800 \mathrm{ng} / \mathrm{ml}$ ) with normal iron stores. Stool culture was negative.

A bone marrow biopsy and aspirate smears showed a hypercellular marrow with erythroid hyperplasia, megaloblastic erythroblasts, giant bands and metamyelocytes (fig. 1) and normal iron stores, findings classic for megaloblastic anemia. Upper endoscopy performed for evaluation of possible gastrointestinal bleeding and malabsorptive disease revealed mildly atrophic gastric mucosa and normal appearing small bowel mucosa including the proximal jejunum. Biopsy of the stomach showed chronic active Helicobacter pylori (H. Pylori) -related gastritis. Jejunal biopsies showed moderate villous atrophy with increased intraepithelial lymphocytes (IELs, fig. 2A\&B). A diagnosis of coeliac disease was suggested based on the jejunal biopsy findings.

Coeliac serologies (tissue transglutaminase IgA antibody, anti-gliadin $\operatorname{IgA}$ antibody) were negative. A subsequent colonoscopy with terminal ileum biopsies showed mild to moderate villous blunting with increased IELs. Biopsies of the colon were normal. Based on the negative coeliac serologies and terminal ileum findings as well as severe macrocytic anemia, a diagnosis of TS was suggested.

The macrocytic anemia quickly responded to vitamin $\mathrm{B}_{12}$ and folic acid supplements after three weeks with a corrected MCV from 108.5 to $81 \mathrm{fL}$ and $\mathrm{Hb}$ to $9.7 \mathrm{~g} / \mathrm{dl}$. Appropriate tripleantibiotic therapy was administered for $\mathrm{H}$. pylori infection. Four months after initial presentation, all symptoms had resolved and hemoglobin had rebounded to $13.2 \mathrm{gm} / \mathrm{dL}$ with MCV $80.3 \mathrm{fL}$. A repeat upper endoscopy with repeat duodenal biopsies showed improved, but ongoing pathology marked by milder villous shortening and persistent IELs.

Table 3: Comparison of the findings of this study with other studies

\begin{tabular}{lll}
\hline & Coeliac sprue & Tropical sprue \\
\hline Geography & $\begin{array}{l}\text { Mostly Europe \& North America, parts of Northern } \\
\text { India }\end{array}$ & Indian subcontinent, West Africa, Caribbean \\
Etiology & Autoimmune, wheat gluten & Hypoplastic anemia \\
\hline Genetic:HLA-DQ2, DQ8 & Infection, aerobic enteric bacteria & Hypoplastic anemia(40.6\%) \\
Serology & ELISA for tissue transglutaminase & None \\
Iron deficiency anemia & +++ & Rare \\
Vitamin B12, folate deficiency & + & +++ \\
Distribution & Proximal duodenum, jejunum & Entire small intestine including ileum \\
Histology & Villous atrophy-none to flattened (Marsh 1-3) & Total villous atrophy -rare \\
& $\begin{array}{l}\text { Crypt hyperplasia } \\
\text { Lamina propria- increased chronic inflammation }\end{array}$ & Lamina propria- increased chronic inflammation \\
& Increased IELs & Increased IELs \\
\hline
\end{tabular}


Due to ongoing small bowel findings, a month-long course of tetracycline was started for a presumed diagnosis of TS. A third upper endoscopy after the month-long antibiotic course showed resolution of duodenal villous atrophy and increased IELs (fig. 3).

\section{DISCUSSION}

Malabsorption can be caused by a variety of small intestinal disorders and comprises of a constellation of symptoms that include diarrhea, steatorrhea, and secondary changes such as weight loss, and nutritional deficiency including iron, folate and vitamin deficiencies from reduced absorption. TS is a rare disease of unknown etiology marked by malabsorption, nutritional deficiencies, and mucosal abnormalities of the small bowel, occurring among residents of or visitors to tropical countries. ${ }^{1}$ TS has been known since the ancient times being described in the ancient Indian medical treatise, Charaka samhita over two thousand years ago. ${ }^{1}$ The diagnosis appears to have a geographic focus affecting population living along a narrow band of landmass near the equator. The diagnosis is made almost exclusively in people from India, Southeast Asia, and the Caribbean islands, yet appears to spare populations in Jamaica, the Bahamas, and sub- Saharan Africa. ${ }^{4}$

In addition to the indigenous population, visitors who spend at least one month in these areas are at risk for developing TS. ${ }^{1}$ Exposure is generally followed by an acute diarrheal illness, but TS can remain as a latent process for many years even after an expatriate has returned to their country of origin as was the case with our patient.

It has been reported that TS is on the decline with fewer documented cases in the United States and Caribbean. Explanations for the decreasing incidence include the improvement of identifying celiac sprue through more accurate serologic testing, widespread use of antibiotics that may treat undiagnosed TS disease and improved sanitation. ${ }^{5}$ TS may be on the decline in India as well. ${ }^{6}$

The etiology of TS remains unclear but an infectious cause is believed most likely due to its response to antibiotics. Although no single infectious organism has been identified, enterotoxigenic coliforms such as Escherichia coli, Klebsiella pneumoniae and Enterobacter cloace have been isolated in patients with TS. ${ }^{7}$ It is speculated that bacterial overgrowth leads to a disturbance in gut motility causing small bowel stasis, resulting in damaged enterocytes. As a consequence, malabsorption with folate loss ensues and the continued loss of folate hinders enterocyte recovery. ${ }^{8,9}$

Patients with TS typically present with progressive weight loss and chronic diarrhea with associated steatorrhea, anorexia, abdominal cramps, and bloating. Over months to years, a malabsorptive syndrome can manifest as glossitis, stomatitis, and pedal edema. Macrocytic anemia will develop initially from the malabsorption of folate with Vitamin $B_{12}$ deficiency

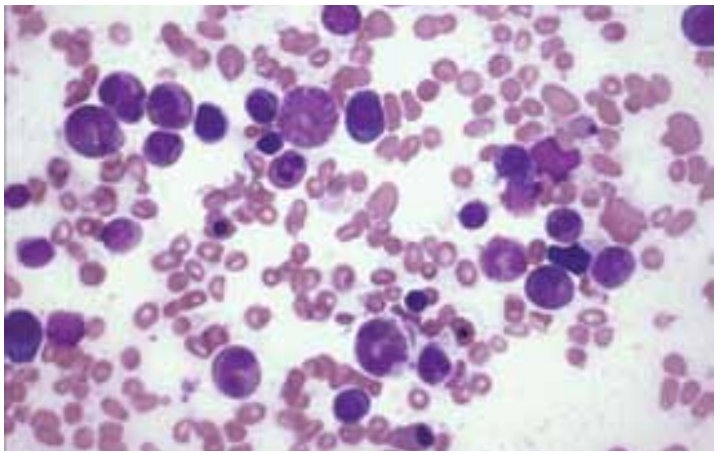

Figure 1: Hypercellular bone marrow aspirate smear showing erythroid hyperplasia, giant metamylocytes and band cells (Wright stain, X400).
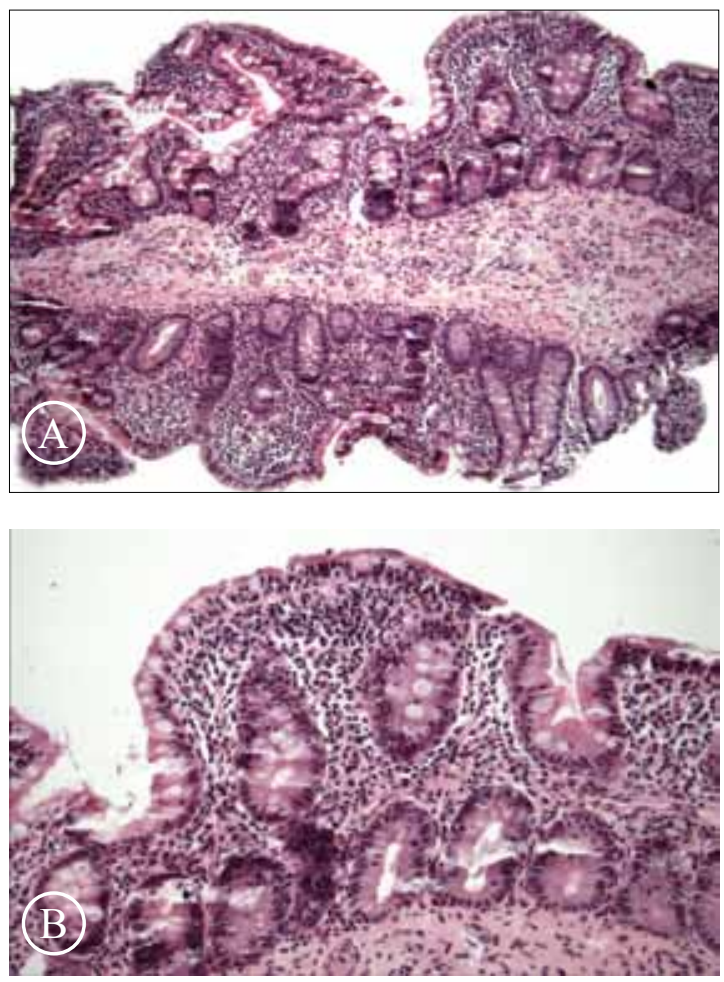

Figure $2 A$ and B: Jejunal biopsy showing villous atrophy with increased Intraepithelial lymphocytes ( $H E$ stain, X100).

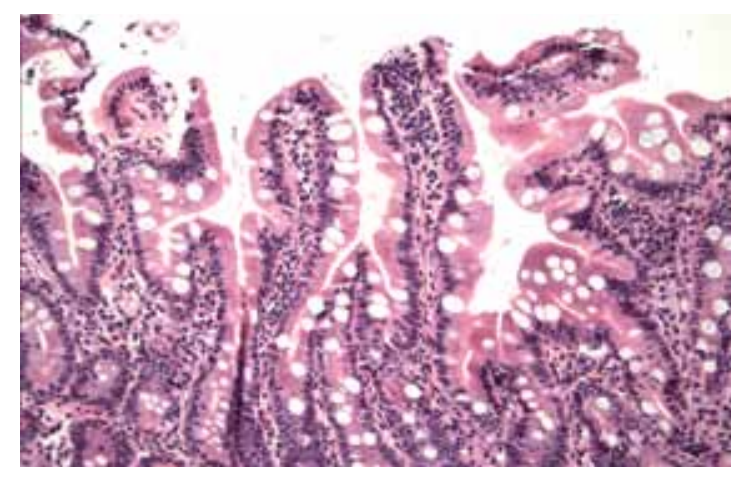

Figure 3: Duodenal biopsy showing resolution of villous atrophy after a month of treatment (HE stain, X100). 
often following from terminal ileum involvement after a prolonged course of the disease. Mucosal changes in TS can involve the entire small bowel with the jejunum and ileum equally affected by the injurious process. ${ }^{10}$ Endoscopically TS can resemble coeliac with mucosal scalloping. The mucosal changes seen include crypt elongation, villous blunting and mononuclear cell infiltration of the lamina propria and epithelium. $^{10,11}$ In contrast, Coeliac disease is characterized by proximal small bowel involvement, not surprising since the highest concentration of dietary gluten is present in the duodenum and jejunum. The biopsy protocol for diagnosis of malabsorption is shown in Table 2. The comparison between Coeliac and non coeliac sprue is shown in Table 3.

It is this difference in the length of bowel involvement that results in severe $B_{12}$ and folate deficiency in TS. The flattened duodenal mucosa that is common in coeliac is not seen in TS where the villi are mild to moderately blunted. Megalocytosis of crypt epithelium can be present and should not be a concern for dysplasia. Treatment with folic acid alone can be curative in those who have TS for only a few months. ${ }^{12}$ Antibiotics have been used since the 1930's for the treatment of TS. Tetracycline therapy (1 gram per day) for one month or up to 3-6 months in endemic areas has been shown to restore the intestinal mucosa in a majority of patients. ${ }^{13}$ Recurrence of TS after completing a course of antibiotics can be seen in up to $20 \%$, especially those who remain in endemic areas. ${ }^{14}$ Following a one month course of tetracycline, a repeat duodenal biopsy in our patient showed normal duodenal mucosa with resolution of villous blunting. Elimination of bacterial overgrowth by tetracycline and mucosal repair by folate supplements result in the restoration of mucosal integrity. ${ }^{8}$ Parenteral vitamin $B_{12}$, oral folate and iron replacement result in prompt resolution of megaloblastic anemia, glossitis, anorexia and weight gain even before improvement in intestinal absorption. ${ }^{15}$

\section{CONCLUSION}

We report a case of TS that appeared to be consistent with latent TS occurring 5 years after the most recent presumed exposure in the Dominican Republic. Our patient displayed classic findings of advanced TS with severe folate and Vitamin $\mathrm{B}_{12}$ depletion in concert with proximal and distal small bowel pathology. The presumed diagnosis was also supported by prompt response to folate and histologic resolution with a month-long antibiotic course.

\section{REFERENCES}

1. Ramakrishna BS, Venkataraman S, Mukhopadhya A. Tropical malabsorption. Postgrad Med J 2006;82:779-87.

2. Baker SJ, Klipstein FA. Regarding the definition of tropical sprue. Gastroenterology 1970;58:717-21.

3. Walker MM. What is Tropical Sprue? J Gastroenterol Hepatol 2003; 18:887-90.

4. Lim ML. A perspective on tropical sprue. Curr Gastroenterol Rep 2001;3:322-7.

5. Tomkins A, Booth CC. Tropical sprue. In: Disorders of the Small Intestine. Oxford, Blackwell Scientific Publications: Disorders of the Small Intestine. Oxford, Blackwell Scientific Publications, Boston; 1985. pp311-32.

6. Bartholomew C. William Hillary and sprue in the Caribbean: 230 years later. Gut 1989;30:17-21.

7. Thakur B, Mishra P, Desai N, Thakur S, Alexander J, Sawant P. Profile of chronic small bowel diarrhea in adults in Western India: a hospital based study. Trop Gastroenterol 2006;27:84-6.

8. Klipstein FA, Engert RF, Short HB. Enterotoxigenicity of colonizing coliform bacteria in tropical sprue and blind-loop syndrome. Lancet $1978 ; 2: 342-4$.

9. Cook GC: Aetiology and pathogenesis of post infective tropical malabsorption (tropical sprue). Lancet 1984;1:721-3.

10. Ghoshal UC, Ghoshal U, Ayyagari A et al. Tropical sprue is associated with contamination of small bowel with aerobic bacteria and reversible prolongation of orocecal transit time. J Gastroenterol Hepatol 2003;18:540-7.

11. Wheby MS, Swanson VL, Bayless TM: Comparison of ileal and jejunal biopsies in tropical sprue. Am J Clin Nutr 1971;24:117-23.

12. Bayless TM, Swanson VL, Wheby MS: Jejunal histology and clinical status in tropical sprue and other chronic diarrheal disorders. Am J Clin Nutr 1971;24:112-6.

13. Sheehy TW, Baggs B, Perez-Santiago E, Floch MH: Prognosis of tropical sprue. A study of the effect of folic acid on the intestinal aspects of acute and chronic sprue. Ann Internal Med 1962;57:892908.

14. Jeejeebhoy KN, Desai HG, Noronha JM, Anita FP, Parekh DV. Idiopathic tropical diarrhea with or without steatorrhoea (tropical malabsorption syndrome). Gastroenterology 1966;51:333-44.

15. Rickles FR, Klipstein FA, Tomasini J, et al. Long-term follow up of antibiotic-treated tropical sprue. Ann Internal Med 1972;76: 203-10.

16. Tomkins AM, Smith T, Wright SG. Assessment of early and delayed responses in vitamin B12 absorption during antibiotic therapy in tropical malabsorption. Clin Sci Mol Med Suppl 1978;55:533-9. 